\title{
The Optimization Design of the Four-bar linkage Based on MATLAB
}

\author{
Jianxia Li \\ 118mail box, The First Aeronautic Institute of Air Force , Xinyang city,Henan Prov.464000 \\ gzwljx@sohu.com
}

Keywords: MATLAB; the four-bar linkage; optimization design

\begin{abstract}
This paper introduces the usage method of the MATLAB optimization toolbox and the application of MATLAB software to solve the problems in mechanical optimal design. By analyzing, the mathematical model of optimum design of the four-bar linkage is proposed, and the optimization design of four-bar linkage is realized by the MATLAB optimization toolbox,The method and step of the mechanical optimized design are introduced.
\end{abstract}

\section{Introduction}

Mechanical optimum design is a new design procedure based on the development of the theory of modern design. It is applied widely in the mechanical component design. When a certain machinery product is optimized, the best parameters are chose by the prescriptive constraint conditions, which make a design index or several indexes obtain the optima value. In optimization, we should first build mathematical model of engineering design, then select optimization method and corresponding calculating program according to the characteristics of the model, at last obtain the optimal design by computer.

Four-bar linkage is one of the important parts of mechanical drive system. It is usually designed by drawing or experimentation. Both methods are simple, but can cause large error. The analytic method can accord with the design demand, but the manual process is extremely complex, even is not achieved. The optimum design method of four-bar linkage based on MATLAB optimization toolbox can simplify computer programming and the input of initial parameters, and also can meet the requirement with speed and accuracy. There are many types of four-bar linkage. With the example of rank-and- rocker mechanism on the designated track, the optimum design method is introduced here.

\section{The machine optimization design method based on MATLAB}

MATLAB language is a software of engineering calculation with rich MATLAB optimization toolbox in it. MATLAB optimization toolbox has several optimal methods in common use, and it is widely used in some problems, such as linear programming, quadratic programming, nonlinear optimization problem, least square method, nonlinear equation, multi-target decision and so on. The function is simple, the optimization algorithm is selected flexibility, the parameters are set freely, and it has evident superiority contrasted with some other mature optimized procedures.

MATLAB optimization toolbox is a powerful engineering calculation program. It incorporates various functions including number calculation, the symbolic computation, visual modeling, simulation and graphic processing. It is widely applied in many engineering fields, such as mechanical design, automatic control and the mathematical statistics. The engineering problem is solved by MATLAB optimization toolbox, and the process is summarized as following:

(1) According to the problem, optimization mathematical model are built, variable, constraint condition and objective function are defined.

(2) Analyze the built mathematics model, select the appropriate optimization method, call the corresponding function in MATLAB optimization toolbox.

(3) Complete the optimized computation and analyze the optimized results. 
The key problems are to build mathematics model and whether the built mathematics model is good, which will directly determine whether the optimized result is effective.

\section{Build the optimization design model of four-bar linkage on the designate track}

Define design variables. The four-bar linkage on the designated track is that the coupler curve of the mechanism is as close as possible to a given curve line. When the crank $l_{1}$ moves with constant speed, the rocker $l_{3}$ is required to move by the known law $\psi_{E}(\varphi)$.The length of the rod and the initial position (angle) of rocker are design variables.

$$
x=\left[\begin{array}{lllll}
x_{1} & x_{2} & x_{3} & x_{4} & x_{5}
\end{array}\right]^{T}=\left[\begin{array}{llllll}
l_{1} & l_{2} & l_{3} & l_{4} & \varphi_{0}
\end{array}\right]^{T}
$$

In counting, usually $l_{1}$ adopts 1 , and the length of other rod is a multiple of $l_{1}$ at the rate. The initial position (angle) of the crank and the rocker $l_{3}$ are functions of the length. $l_{2}, l_{3}, l_{4}$ are independent variables and the design variables are:

$$
x=\left[\begin{array}{lllll} 
& x_{2} & x_{3} & x_{4}
\end{array}\right]^{T}=\left[\begin{array}{llllll}
l_{2} & l_{3} & l_{4}
\end{array}\right]^{T}
$$

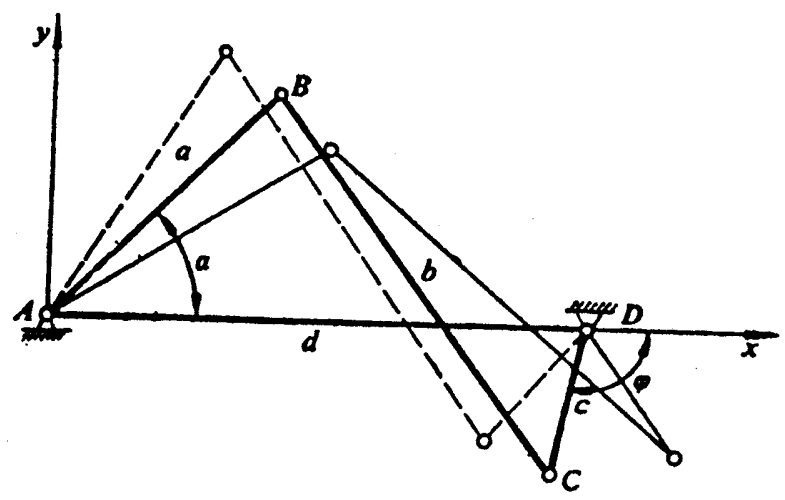

Fig.1 Four-bar linkage

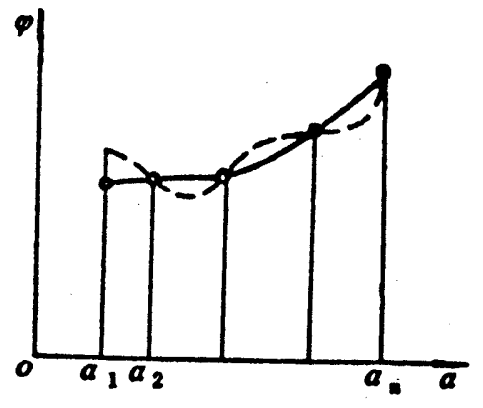

Fig.2 The target function

Establish objective function. If the four-bar linkage can motion as the given continuous function $\psi_{E}(\varphi)$, that is to say, when the driven part $\mathrm{AB}$ is at position $\alpha_{1}, \alpha_{2}, \alpha_{3}, \ldots, \alpha_{m}$, the follower is at position $\varphi_{1}, \varphi_{2}, \varphi_{3}, \ldots, \varphi_{m}$ accordingly, Three sets of values of $\alpha$ and the corresponding $\varphi$ can decide a four-bar linkage(Fig.1), In order to make the selected size parameters satisfy the motion relation demanded approximately and reduce the absolute values of deviations at most, the appropriate motion curve of the linkage is set according to the relevant position of the driving part and the follower, and the target function is built by the least square method as shown in Figure 2. Solid line indicates expectant motion law of mechanism, the dotted line indicates the appropriate motion curve which is built by the least square method.

Set up the constraints. Crank-rocker mechanism shall satisfy the existing conditions of crank, and at the same time, the transmission angle should be between $\gamma_{\min }$ and $\gamma_{\max }$, so the transmission conditions are:

$$
\begin{aligned}
& g_{1}(x)=l_{1}-l_{2} \leq 0 \\
& g_{2}(x)=l_{1}-l_{3} \leq 0 \\
& g_{3}(x)=l_{1}-l_{4} \leq 0 \\
& g_{4}(x)=l_{1}+l_{4}-l_{3}-l_{4} \leq 0
\end{aligned}
$$




$$
\begin{aligned}
& g_{5}(x)=l_{1}+l_{2}-l_{3}-l_{4} \leq 0 \\
& g_{6}(x)=l_{1}+l_{3}-l_{2}-l_{4} \leq 0 \\
& g_{7}(x)=\arccos \left[\frac{l_{2}^{2}+l_{3}^{2}-\left(l_{1}+l_{4}\right)^{2}}{2 l_{2} l_{3}}\right]-\gamma_{\max } \leq 0 \\
& g_{8}(x)=\arccos \left[\frac{l_{2}^{2}+l_{3}^{2}-\left(l_{4}-l_{1}\right)^{2}}{2 l_{2} l_{3}}\right] \leq 0
\end{aligned}
$$

\section{Realize the Optimization Design of Four-bar Linkage by MATLAB}

According to the optimization objective functions and constraint conditions, this is a optimization design problem with three design variables and eight inequality constrains. We can get the result by penalty function.

In the MATLAB optimization toolbox, there are many functions which are used to solve constrained optimization problems: fminbnd(scalar bounded nonlinear function minimization) 、 fmincon (constrained nonlinear minimization) 、 fseminf(constrained minimization, semi-infinite constraints) 、quadprog (quadratic programming) 、fminmax (minimax optimization) . Fmincon is choosen in this paper.

The following is fmincon's format:

$[\mathrm{x}, \mathrm{fval}]=$ fmincon (fun,x0,A,b,Aeq,beq,lb,ub,nonlcon,options,P1,P2.....)

When we use fmincon, objective function should be defined, .m file should be called and the known parameters should be inputted, such as coefficient matrix and constraint vector of linear inequality constraints of initial point, coefficient matrix and constraint vector of linear equality constraints, lower bound vector and upper bound vector and so on. The optimal solution of the objective function and it's value are outputted after the program runs.

Write M-file of the objective function (myfun.m)

function $\mathrm{f}=$ myfun $(\mathrm{x})$

$\mathrm{g}=\left(\psi_{\mathrm{Ei}}-\psi_{\mathrm{i}}\right)^{\wedge 2}$

$\mathrm{f}=\operatorname{symsum}(\mathrm{g}, \mathrm{m}+1)$

Write M-file of the constraint function (myfun.m)

funciton[h,geq ] =mycon(x)

According to the constraints that have been defined, we can write geq=[ ].

Call the optimizer in the command window. Inputted Initial data of the optimization design in the command window, call the function

[l fval]=fmincon(' mycon l0,A,b,Aeq,beq,lb,ub,'mycon'), then we can get the optimization results. It is verified that the optimal point is at the intersection of all constraints.

\section{Conclusion}

The optimum design method of four-bar linkage based on MATLAB optimization toolbox is introduced in this paper, and the result shows we get the predefined effect. MATLAB optimization toolbox can realize the procedure of mechanical optimum design well, make the solution quicker and the procedure easier, improve efficiency, so it has great advantage and is important tool to solve the problems in mechanical optimal design. But optimum design method of the four-bar linkage is synthesis optimization question of multiple constraints and multi-variable type. The length of rod is only considered as design variable in this paper, of course, more design variables can be considered in order to improve the accuracy, but proportionately, it also can increase the computation and complexity. Study shows many optimization problems in the engineering projects, especially the structural parameters optimization, are solved easily and quickly by the MATLAB optimization 
toolbox.

\section{References}

[1] Sun Jingmin: Optimization of Mechanical Design ( Harbin Institute of Technology Press, Harbin 2004). In Chinese.

[2] Zhang Shengqin: MATLAB 7.0 practical guide ( Mechanical Industry Press, Beijing 2008). In Chinese. 